\title{
Observation of electron-transfer-mediated decay in aqueous solution
}

\author{
Isaak Unger ${ }_{1+}$, Robert Seidel, Stephan Thürmer ${ }_{1+}$, Marvin N. Pohl ${ }_{1}$, Emad F. Aziz1,2,3, \\ Lorenz S. Cederbaum4, Eva Muchová5, Petr Slavíček ${ }^{*}$, Bernd Winter ${ }^{*}$ and Nikolai \\ V. Kryzhevois ${ }^{*}$ \\ Helmholtz-Zentrum Berlin für Materialien und Energie, Institute of Methods for Material Development, Albert-Einstein-Strasse \\ 15, D-12489 Berlin, Germany. \\ ${ }_{2}$ Department of Physics, Freie Universität Berlin, Arnimallee 14, D-14159 Berlin, Germany. \\ ${ }_{3}$ School of Chemistry, Monash University, Victoria 3800, Australia. \\ 4Theoretische Chemie, Physikalisch-Chemisches Institut, Universität Heidelberg, Im Neuenheimer Feld 229, D-69120 \\ Heidelberg, Germany. \\ ${ }_{5}$ Department of Physical Chemistry, University of Chemistry and Technology, Technická 5, 16628 Prague, Czech Republic; \\ †Present addresses: Department of Physics and Astronomy, Uppsala University, Box 516, SE-751 20 Uppsala, Sweden (I.U.); \\ Department of Chemistry, Kyoto University, Kitashirakawa-Oiwakecho, Sakyo-Ku, Kyoto 606-8502, Japan (S.T.).
}

*e-mail: petr.slavicek@vscht.cz; bernd.winter@helmholtz-berlin.de; nikolai.kryzhevoi@pci.uni-heidelberg.de

Photoionization is at the heart of X-ray photoelectron spectroscopy (XPS), which gives access to important information on a sample's local chemical environment. Local and non-local electronic decay after photoionization-in which the refilling of core holes results in electron emission from either the initially ionized species or a neighbour, respectively-have been well studied. However, electron-transfer-mediated decay (ETMD), which involves the refilling of a core hole by an electron from a neighbouring species, has not yet been observed in condensed phase. Here we report the experimental observation of ETMD in an aqueous $\mathrm{LiCl}$ solution by detecting characteristic secondary low-energy electrons using liquid-microjet soft XPS. Experimental results are interpreted using molecular dynamics and high-level ab initio calculations. We show that both solvent molecules and counterions participate in the ETMD processes, and different ion associations have distinctive spectral fingerprints. Furthermore, ETMD spectra are sensitive to coordination numbers, ionsolvent distances and solvent arrangement.

Site-selectivity and sensitivity to the local chemical environment have made X-ray photoelectron spectroscopy (XPS) a powerful tool for probing both gas phase and condensed matter-its development has also led to a deeper understanding of the complex and competitive processes that occur as a result of X-ray-substrate interactions. The creation of deep inner-shell electron holes through X-ray photoionization is followed by relaxation processes that provide additional important insight into electronic structure and correlation in the valence-electron region. One such process is Augerelectron decay, in which, within the same initially ionized species, a valence electron relaxes to fill a core vacancy, causing the emission of an electron from a higher state. Auger processes have found widespread applications in many areas of research, especially in materials science and surface-composition analysis. Element-selectivity of these X-rayinduced de-excitations opens a way for targeted energy deposition, which can be used in medicine, in particular for cancer treatment 1 or for selective transformations of molecules and materials2.

However, Auger decay is not the only relaxation process that can occur after initial photoelectron emission. Several experimental and theoretical works have demonstrated electronic relaxation processes that can efficiently compete with local Auger decay and that are 'non-local' in nature, that is, they involve species other than the initially ionized monomer. The best studied process is intermolecular Coulombic decay (ICD)3, which occurs in weakly interacting systems such as rare gases and hydrogen-bonded complexes4,5. In an ICD process, the energy gained after refilling the initial hole created by ionization or excitation is used to eject an electron from a neighbouring species, resulting in the formation of two singly charged units that subsequently separate by Coulomb repulsion. The competition of non-local and local relaxation processes has been recently examined in various aqueous solutions6-8. ICD in an aqueous environment is particularly important because of the production of slow electrons and water radical cations 9,10 that can cause damage to biological tissue.

Here, we address a different and more complex non-local electronic relaxation process, electron-transfer-mediated decay (ETMD) 11 , which remains largely unexplored. The first step in ETMD, unlike in Auger decay and ICD, is the refilling of the created vacancy by an electron from a neighbouring atomic or molecular monomer. The energy released is used to ionize either the same electron-donating monomer $(\operatorname{ETMD}(2)$ process, where $(2)$ refers to the number of species involved in the process), or it is used to ionize a third monomer in the vicinity (ETMD(3) process), thus producing charges that are not located on the initially ionized species (Fig. 1). As a mechanism for the production of dications, ETMD is found to be very efficient. Compared with direct double photoionization, having rather small cross-sections for most molecules, the efficiency of ETMD for double ionization can be larger by several orders of magnitude 12 . This electronic decay mechanism can be utilized to produce ultracold molecular dications that can hardly be produced otherwise, for instance, by trapping the molecules in helium droplets. ETMD thus opens a potential new route for ultracold chemistry13. But so far, ETMD has only been observed experimentally in rare-gas clusters14-16 and in doped 
helium droplets13. These complexes were also in the focus of most of the theoretical ETMD studies (see, for example, refs 12,17,18). ETMD is, however, a general phenomenon, and has been predicted theoretically to occur in other environments, in particular in hydrogen-bonded complexes8,19,20. In fact, the relatively short intermolecular distances and large molecular overlaps in hydrogen-bonded systems are favourable for ETMD.

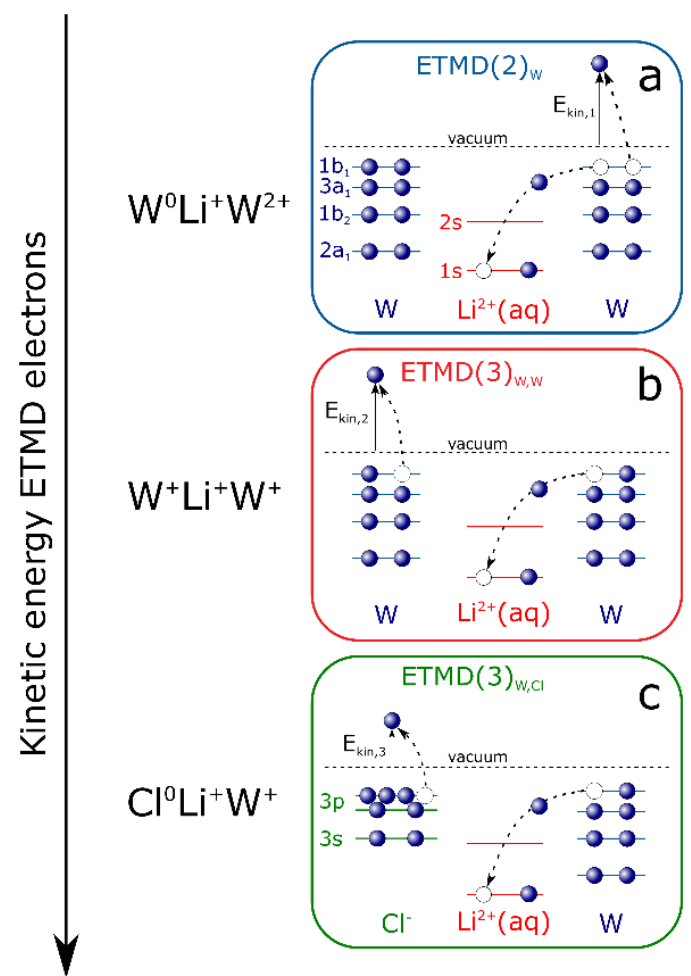

final state core-ionized state
Figure 1 The most relevant ETMD processes in $\mathrm{LiCl}$ aqueous solution. a, ETMD(2)w. b, ETMD(3)w,w. c, ETMD(3)w,cl. The subscripts W and Cl refer to the species ionized in the final state (water molecules and a chloride anion), and (2) and (3) refer to the numbers of monomers involved in the ETMD processes, including the initially core-ionized $\mathrm{Li}_{+}$. The starting point in each case is the 1 s core-level ionization of $\mathrm{Li}^{+}$(aq.) forming $\mathrm{Li}^{2+}$ (aq.). Ekin denotes the kinetic energies of electrons emitted in ETMD processes (briefly ETMD electrons), which are measured in the experiment. The respective final ETMD states and the relative kinetic energies of the ETMD electrons are shown on the left side.

In the present work we report the experimental detection of ETMD in an aqueous solution and show experimentally that it occurs upon core-level ionization; theoretical calculations are used to explain the measured ETMD spectra. In our quest for an experimental spectral signature we chose a system in which no other nonradiative relaxation but ETMD is allowed, and thus the emitted ETMD electrons can be unambiguously assigned. Aqueous $\mathrm{LiCl}$ solution is particularly suitable, and also the simplest candidate. Core-ionized $\mathrm{Li}_{+}$(aq.) cannot decay electronically via Auger or ICD mechanisms since $\mathrm{Li}+$ has no valence electrons. ETMD is possible, however, and proceeds quickly, within $20 \mathrm{fs}($ ref. 19). Yet the detection of ETMDelectrons is experimentally challenging. Current detection techniques require long acquisition times and a stable liquidmicrojet position in order to identify the ETMD signal on the large background from secondary (inelastically scattered) electrons forming the low-kinetic energy part of the photoelectron spectrum. In order to explain more specifically what our study is expected to reveal, we illustrate in the energy-level diagrams of Fig. 1 the most relevant $\operatorname{ETMD}(2)$ and $\operatorname{ETMD}(3)$ processes following 1s ionization of Li+(aq.); other ETMD processes are not shown in the figure but will be considered in our calculations. The $\operatorname{ETMD}(2)$ w process shown in Fig. 1a involves a single water molecule $(\mathrm{W})$, which donates a valence electron to fill the $\mathrm{Li}_{2+}$ (aq.) core hole. The released energy is then used to ionize another valence electron from the same water molecule. In the experiment, the kinetic energy of this latter electron, ejected into vacuum, is detected. Contrary to $\operatorname{ETMD}(2) w$, in $\operatorname{ETMD}(3) w, w(F i g . ~ 1 b)$ the energy released in the first electron-transfer step is used to ionize a second water molecule so that two different water molecules become ionized in the end. In the presence of a counter ion, $\mathrm{Cl}$ - in this study, the energy released in the first ETMD step can be also transferred to this anion, causing electron detachment. The respective $\operatorname{ETMD}(3) \mathrm{w}, \mathrm{cl}$ process where the subscripts refer to the species ionized in the final state is depicted in Fig. 1c.

Apparently, the electrons emitted in different ETMD processes have different kinetic energies thus leading to characteristic spectral shapes depending on the specific local atomic environment. This is indeed confirmed by experimental spectra that exhibit several features in the energy distribution of ETMD electrons. The structured ETMD spectrum can be used to infer local solvation-structure details, especially various forms of ion pairing (solvent-separated, contact or solvent-shared ion pairs). The potential of this latter aspect is demonstrated here in some depth by the example of $\mathrm{Li}_{+}$(aq.) and also on the base of electronic structure calculations for a few simple model configurations of $\mathrm{Li}_{+}$solvation. Our goal is to convey that the ETMD process is not just operating in aqueous solutions but that it can also be used to generate radicals and lowenergy electrons within a small tunable energy window and independent of the applied ionization energy, factors that play an important role in radiotherapy.

\section{Results and discussion}

Experimental spectra. ETMD spectra from 3 and $4.5 \mathrm{M} \mathrm{LiCl}$ aqueous solutions, measured at 171 and $175 \mathrm{eV}$ photon energies, respectively, are shown in tiers $\mathrm{b}$ and $\mathrm{c}$ of Fig. 2. The photon energies applied are well above the $\mathrm{Li}_{+}(\mathrm{aq}$. ionization threshold $\left(60.4 \mathrm{eV}_{21}\right)$ in water. Energies of the measured electrons are presented as kinetic energies (top scale) and as double-ionization energies of the final states (bottom scale). We refer to the latter scale in the following discussion. Spectra $b$ and $c$ are obtained from subtraction of a neat-water photoelectron spectrum from the respective solution spectrum. As an example, in Fig. 2a we show the data for $3 \mathrm{M}$ concentration, displayed over the range of relevant electron kinetic energies. With respect to the neat-water spectrum (blue) a small signal increase near $30 \mathrm{eV}$ kinetic energy, which will be assigned to ETMD electrons, is observed in the solution spectrum (red). Note that both spectra in Fig. 2a are dominated by the emission of inelastically scattered (photo) electrons, which give rise to the characteristic rather 


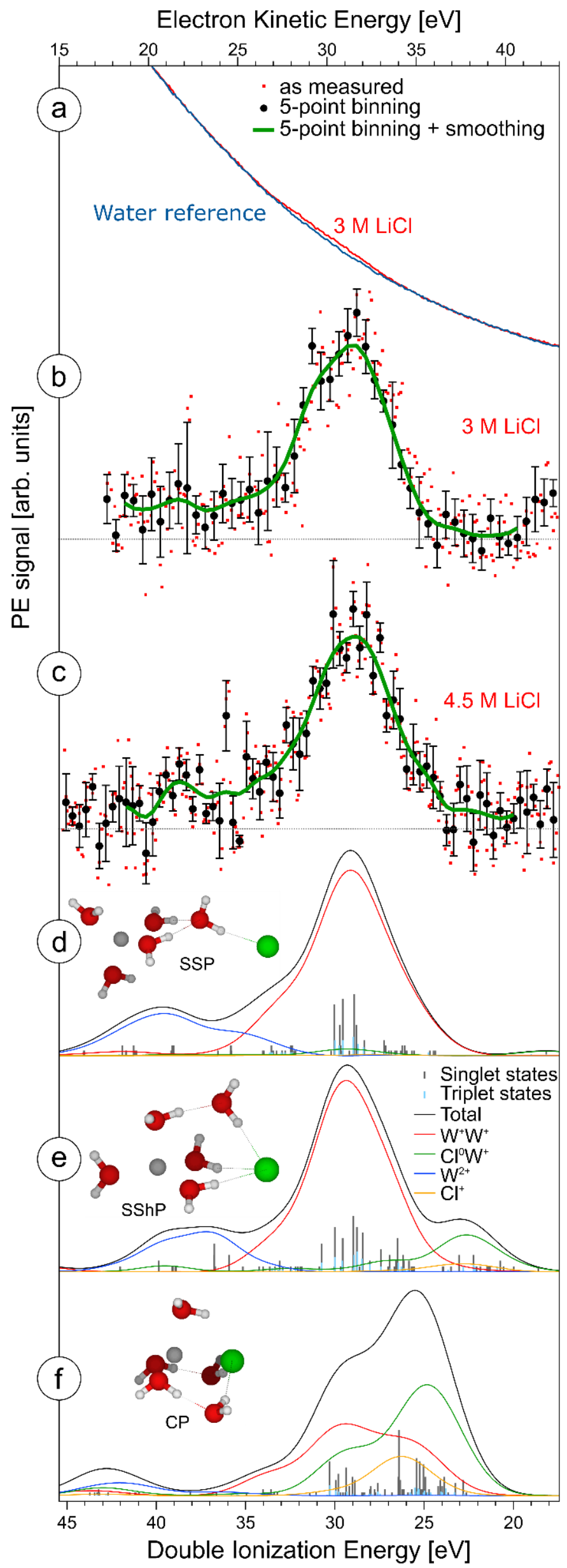

structure-less large electron signal steadily increasing towards lower kinetic energies. Since the ETMD signal of interest is much smaller than the background signal from inelastic electrons, the differential spectra (Fig. 2b,c) exhibit rather low signal-to-noise ratios. In order to show that the data are statistically significant the as-measured individual data points have been binned and the resulting error bars have been determined. Results for five-point binning are presented by the black full circles, and additional smoothing yields the green line. As detailed in the Methods section 'Experimental' better statistics cannot be achieved for the ETMD spectra with the present setup using standard electronenergy detection schemes. Future electron-electron-coincidence measurements will deliver higher-quality spectra. Such an experimental approach has been recently demonstrated for the inner-valence ionization of water clusters, where ICD electrons could be distinguished from the direct photoelectrons 10 .

Figure 2 | Experimental and simulated ETMD spectra of $\mathrm{LiCl}$ aqueous solutions. All ETMD spectra are shown on the kinetic energy (top) and double-ionization energy (bottom) scales. a,b, Experimental ETMD spectra from 3.0 M concentration resulting from core ionization of $\mathrm{Li}_{+}$ (aq.) at $171 \mathrm{eV}$ photon energy. a, The as-measured spectrum of the $\mathrm{LiCl}$ aqueous solution (red) and the reference spectrum of neat water (which only contains contributions from inelastically scattered photoelectrons; blue). b, The resulting difference spectrum, solution minus water, yielding the red dots. Black dots result from five-point-binning of the red dots, and the green line results from additional smoothing. $\mathrm{c}$, The analogous data as in b but for $4.5 \mathrm{M}$ concentration, and a photon energy of $175 \mathrm{eV}$. Error bars in b,c represent the standard deviation from five-point-binning. $\mathrm{d}-\mathrm{f}$, Theoretical ETMD spectra (black solid curves) computed for the solvent-separated (SSP), solventshared (SShP), and contact (CP) ion pair cluster models, respectively. Energies and intensities of individual transitions are shown also as sticks. Each stick has been convoluted by a Gaussian with full width at half maximum of $3.6 \mathrm{eV}$. The geometries of the cluster models are depicted in the insets (red: oxygen; green: $\mathrm{Cl}_{-}$; grey: $\mathrm{Li}_{+}$; white: hydrogen). The theoretical ETMD spectra are decomposed into various contributions corresponding to different ETMD processes (coloured solid curves, see the key).

It is seen from Fig. $2 b, c$ that the spectra from both solutions are rather similar, suggesting a small concentration dependence on ion pairing between 3-4.5 M. Furthermore, the fact that the spectral positions are independent of photon energy shows clearly that the signal indeed arises from electronic decay, and contributions from direct ionization can be ruled out. We observe a broad emission structure in the $45-20 \mathrm{eV}$ range, with a dominant peak at 28.5 $\mathrm{eV}$. This peak is attributed to the $\operatorname{ETMD}(3) \mathrm{w}, \mathrm{w}$ processes producing two outer-valence ionized water molecules $\mathrm{H}_{2} \mathrm{O}_{+}\left(3 \mathrm{a}_{1}\right)$. Qualitatively, this can be seen from the consideration of two electrostatically interacting water cations. In the 
case of perfect electronic screening the corresponding ETMD signal would appear at $27 \mathrm{eV}(2 \times 13.50$, where $13.50 \mathrm{eV}$ is the binding energy of the $3 a_{1}$ orbital in liquid water21); close to the experimental value. The small energy difference ( $\sim 1.5 \mathrm{eV}$ ) is probably due to residual repulsion energy that cannot be screened completely in aqueous media. The theoretical calculations described below support this qualitative assignment and provide additional insight into the participating electronic transitions.

Ion pairing in aqueous $\mathrm{LiCl}$ solutions. Aqueous $\mathrm{LiCl}$ solution has been extensively studied, both experimentally and by means of molecular dynamics simulations22-28, and its structure, in particular ion pairing, is well understood. Here, we apply classical molecular dynamics simulations with electronic continuum correction to investigate ion pairing. We show below that the solvent-shared arrangement is expected to prevail in $\mathrm{LiCl}$ solutions in the concentration range studied in our work. Figure 3 presents the radial distribution functions for $\mathrm{Li}_{+}-\mathrm{Cl}-$ and $\mathrm{Li}_{+}-\mathrm{O}$ for aqueous solutions of $3 \mathrm{M}, 4.5 \mathrm{M}$, and also $6 \mathrm{M}$ (for comparison). These results are in good agreement with the data reported previously for salt concentrations in the range 3-6 $\mathrm{m}$ (ref. 22). While the $\mathrm{Li}_{+}-\mathrm{O}$ curves are nearly indistinguishable, the radial distribution function for $\mathrm{Li}_{+}-\mathrm{Cl}$ - varies with concentration. This is particularly clear for the first maximum of this curve at $r=2.36 \AA$ corresponding to a contact ion pair arrangement. However, the second peak at $r=4.62 \AA$, attributed to a solventshared ion pair structure, reveals only minor dependence on concentration. A histogram showing the minimum distance between a selected $\mathrm{Li}+$ cation and the closest $\mathrm{Cl}$ - anion for all three concentrations is depicted in

Supplementary Fig. 1. Quantitatively, the fraction of the contact ion pairs is $17 \%$ for the $3 \mathrm{M}, 27 \%$ for the $4.5 \mathrm{M}$, and $40 \%$ for the $6 \mathrm{M}$ solutions (see Supplementary Fig. 1). Thus, the dominant structure by far is the solvent-shared ion pair structure with $\mathrm{Li}+$ and $\mathrm{Cl}$ - being about $4.5 \AA$ apart; yet, for the $3 \mathrm{M}$ solution, there is still a chance for a solventseparated ion pair. Another way to quantify liquid structure is through coordination numbers. Our results tabulated in Supplementary Table 1 agree reasonably well with other simulations27. As one can see, the chloride anion occupying the first solvation shell becomes a more prominent structure with increasing concentration; yet contact ion pairs are clearly not dominating even for the highest concentration. Another type of structural analysis based on permutation invariant vector clustering that reveals the most populated structural motives is presented in Supplementary Fig. 2.

Cluster models and solvation shifts. Next, we discuss the ETMD spectra. We consider three cluster models: solventseparated ion pairs (SSP), solvent-shared ion pairs (SShP) and contact ion pairs (CP). These models are described in the Methods section 'Computations'. The corresponding spectra are shown in the tiers d, e and f of Fig. 2, respectively. The spectrum of the SShP model is expected to fit best to the experimental data due to prevalence of solvent-shared arrangements in solution. Since the calculations were performed for the gas phase, the spectra need to be shifted in energy to account for solvent effects. The dominant contribution to this shift results from the long-range polarization, which acts differently on different ETMD states. Note that in the photoemission spectra of aqueous electrolytes, the energies of the photoelectron peaks of both the solvent water molecules and the solutes are virtually unaffected by the electrolyte concentration 21 (this is not the case in finite-size systems where the peak positions depend strongly on the local solvation structure, including ion pairing). Apparently, water is capable of very efficiently screening the electrostatic interactions between neighbouring molecules. Thus, we can assume that the energy position of the same ETMD state in the experimental spectra of aqueous solutions does not depend on the environment. In particular, it is not important for the double-ionization energy of two neighbouring water units involved in ETMD(3)w,w whether their environment contains water molecules or ions. Since solvent-shared ion pairs prevail in the measured $\mathrm{LiCl}$ aqueous solutions, and the ETMD(3)w,w processes play the most important role in such structural units (see below), we chose the $\operatorname{ETMD}(3)$ w,w states for the alignment of all spectra. Accordingly, the spectra of the SSP, SShP and CP models were shifted to lower double-ionization energies by 7.65, 5.03 and $3.89 \mathrm{eV}$, respectively. It should be noted that in contrast to the SSP and SShP models, the ETMD(3)w,w processes in the CP model mostly contribute to the high-energy shoulder and not to the main peak, which originates from different processes. Therefore, the main peak in the theoretical spectrum of this model does not coincide in energy with the main peaks in the experimental spectra.

ETMD in solvent-separated and solvent-shared ion pairs. We now discuss the spectral shapes in more detail. The ETMD spectrum of the SShP model exhibits a well-defined main peak at $28.5 \mathrm{eV}$ and two smaller peaks, one spreading between 35 and $40 \mathrm{eV}$ and another one at $22 \mathrm{eV}$. The ETMD spectrum of the SSP model has a similar structure except for the missing peak at the low doubleionization energy side, and a more pronounced shoulder of the main peak at 33 $\mathrm{eV}$. The decomposition of each spectrum into various contributions reveals that the main peak arises essentially from $\operatorname{ETMD(3)w,w~processes.~Interestingly,~only~a~small~fraction~of~the~water~cations~created~in~these~processes~is~found~in~}$ the cationic ground state, that is with the $1 b_{1}$ electron removed. The reason will be discussed below. Most of the water molecules eject electrons from the deeper-lying orbitals during electronic decay. According to our calculations, the main

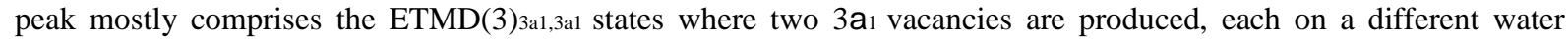
molecule. Its low-energy part at approximately $26 \mathrm{eV}$ is attributed to the $\operatorname{ETMD}(3)_{3 a 1,1 b 1}$ processes creating pairs of $1 b_{1}$ and $3 \mathrm{a}_{1}$-ionized water molecules while the high-energy part at $33 \mathrm{eV}$ corresponds to the processes creating pairs of $1 \mathrm{~b}_{2}$ -

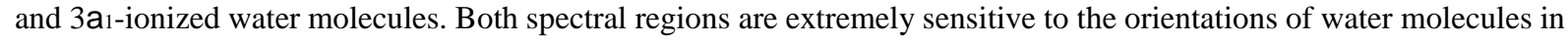
the first solvation shell of the metal ion as seen from Supplementary Fig. 3.

The spectral region near $33 \mathrm{eV}$ also contains some contributions from $\operatorname{ETMD}(2)$ w processes. The main spectral domain of these processes lies however at higher energy, and coincides with the peak that spreads from 35 to $40 \mathrm{eV}$. As for $\operatorname{ETMD}(3) w, w, E T M D(2) w$ also mostly involves $3 a_{1}$ electrons of water molecules, and water dications with two vacancies in the $3 a_{1}$ orbital are the main products of this decay channel. The high probability of the $3 a_{1}$ electrons to participate in 
ETMD in the SShP and SSP models results from favourable orientations of the water monomers in the first solvation shell of $\mathrm{Li}_{+}$. The oxygen atoms of these molecules point towards the cation thus maximizing the overlap of the $3 \mathrm{a}_{1}$ orbitals with the $1 \mathrm{~s}$ orbital of $\mathrm{Li}_{+}$. Although efficiencies of the individual ETMD(3)w,w and ETMD(2)w processes may be comparable, the $\operatorname{ETMD}(3) \mathrm{w}, \mathrm{w}$ peak acquires more intensity. This is because the total $\operatorname{ETMD}(2) \mathrm{w}$ efficiency is approximately proportional to the number of water monomers in the first solvation shell (other water molecules are far less prone to $\operatorname{ETMD}(2)$ ), whereas the total $\operatorname{ETMD}(3)$ w, w efficiency correlates with a much larger number of water pairs, predominantly with one or two water monomers from the first solvation shell. It is also worth mentioning that the relative intensities of the $\operatorname{ETMD}(2)$ w and $\operatorname{ETMD}(3)$ w,w signals are very similar in Fig. 2d and e, which is attributed to similar structures of the first solvation shells of $\mathrm{Li}_{+}$in the respective cluster models. Although the arrangements of water molecules in the immediate neighbourhood of $\mathrm{Li}_{+}$in the SSP and SShP cluster models are very similar, structures differ significantly beyond the first solvation shell of the cation. In the SSP model the chloride anion is far from the metal, appearing only in the third solvation shell. The two counter ions are much closer to each other in the SShP configuration, wherein only two bridging watermolecules separate them. These structural differences are reflected in the ETMD spectra, especially in their low-energy parts where $\operatorname{ETMD}(3)$ w,Cl processes contribute. As can be inferred from Fig. 2, the $\operatorname{ETMD}(3) w, c l$ efficiency depends strongly on the counter-ion separation. In the SShP configuration, the ETMD(3)w,Cl peak at $22 \mathrm{eV}$ is well resolved despite the fact that $\mathrm{Li}+$ and $\mathrm{Cl}$ - are separated by one solvation shell. In the SSP model, this peak is nearly absent.

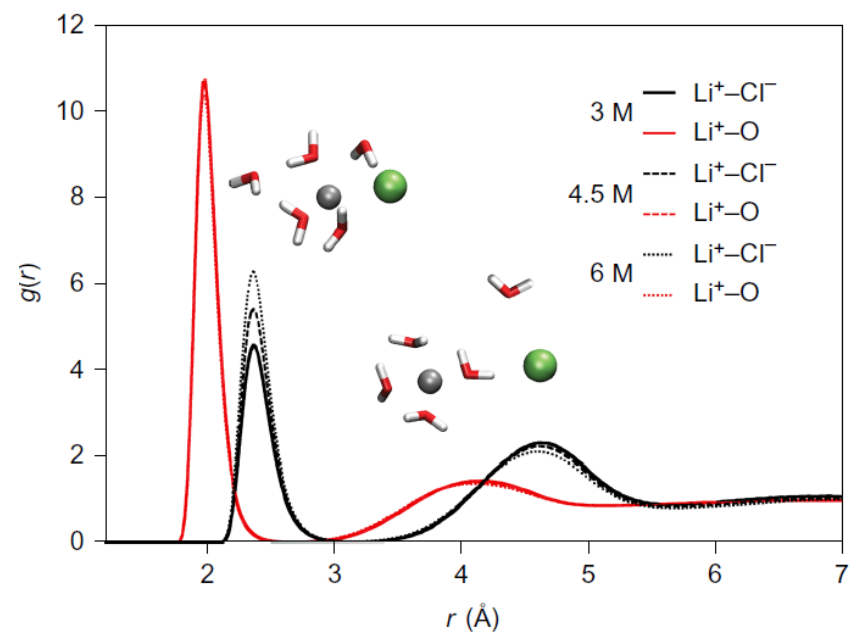

Figure 3 | Radial distribution functions for $\mathrm{LiCl}$ aqueous solutions. Shown are the $\mathrm{Li}_{+}-\mathrm{Cl}-$ and $\mathrm{Li}_{+}-\mathrm{O}$ radial distribution functions for $3 \mathrm{M}, 4.5 \mathrm{M}$ and $6 \mathrm{M} \mathrm{LiCl}$ aqueous solutions. The curves for $\mathrm{Li}_{+}-\mathrm{Cl}$ - vary with concentration, which is particularly well seen for the first maximum at $r=2.36 \AA$ corresponding to a contact ion pair arrangement. The second peak of these curves at $r=$ $4.62 \AA$, attributed to a solvent-shared ion pair structure, reveals however only minor dependence on concentration. The distributions along the $\mathrm{Li}_{+}-\mathrm{O}$ coordinate are nearly indistinguishable. Snapshots from molecular dynamics simulations corresponding to a contact ion pair (left) and a solvent-shared ion pair (right) are shown as insets (grey: $\mathrm{Li}_{+}$; green: $\mathrm{Cl}-$ ).

ETMD in contact ion pairs. The ETMD spectrum changes drastically when one of the water molecules nearest to $\mathrm{Li}+$ is substituted by $\mathrm{Cl}-$, which leads to a contact ion pair. First, the efficiency of $\operatorname{ETMD(3)w,cl~increases~substantially.~The~}$ $\operatorname{ETMD}(3) w, c$ signal, which appears only as a secondary peak in the spectrum of the SShP configuration, now acquires high intensity and becomes the main peak, at $24 \mathrm{eV}$, as seen in Fig. 2f. It should be noted that aside from the ETMD(3)w,Cl contribution, this peak also contains large contributions from the $\operatorname{ETMD}(3) \mathrm{w}, \mathrm{w}$ and $\operatorname{ETMD}(2)_{\mathrm{Cl}}$ processes. The latter produce $\mathrm{Cl}_{+}$cations and seem to appear only in the $\mathrm{CP}$ model. Since $\mathrm{Li}+$ now has less water molecules in the first solvation shell, the impact of the ETMD(3)w,w and ETMD(2)w processes on the total ETMD spectrum decreases. The formation of a contact ion pair also modifies the orientation of water molecules such that the $1 \mathrm{~s}$ orbital of Li+ now better overlaps with the $1 b_{1}$ orbitals of water, and the efficiency of the $\operatorname{ETMD}(3)_{3 a 1,1 b 1}$ processes producing pairs of $1 b_{1-}$ and $3 a_{1-i o n i z e d}$ water molecules increases. At the same time fewer $3 a_{1}$-ionized water pairs are produced by $\operatorname{ETMD}(3)_{3 a 1,3 a 1}$. These trends can be recognized from the red curve in Fig. $2 \mathrm{f}$ whose right and middle peaks originate from the $\operatorname{ETMD}(3)_{3 \mathrm{a} 1,1 \mathrm{~b} 1 \text { and }}$ $\operatorname{ETMD}(3)_{3 a 1,1 b 1}$ processes, respectively; compare with the red curves in Fig. 2d,e.

As seen from Fig. 2, the agreement between experiment and theory is good. The main peak, its shoulder and the peak at higher double-ionization energy are well reproduced and explained by the theory. The situation with the low doubleionization energy region is less clear. By taking into account the above consideration that a certain ETMD state should have the same energy irrespective of the particular environment, and assuming that ETMD(3)w,Cl mostly produces $3 \mathrm{a}_{1-}$ ionized water molecules and neutral chlorines (as predicted by the theory), the corresponding ETMD(3)w,Cl peak should appear at $23.1 \mathrm{eV}(13.5 \mathrm{eV}+9.6 \mathrm{eV}$, where $9.6 \mathrm{eV}$ is the detachment energy of Cl-(aq.)). Arguably, a vaguely visible structure at $22 \mathrm{eV}$ for the $4.5 \mathrm{MLiCl}$ solution can be assigned to this peak. No signal at this energy is, however seen in the spectrum of the 3 Msolution which might be indicative of a larger fraction of solvent-separated ion pairs at this concentration. One needs to perform additional measurements with a higher signal-to-noise ratio to clearly establish experimental spectral differences and prove our assignments.

\section{Conclusions}

We have reported observation of ETMD processes in aqueous media demonstrating that ETMD is a general phenomenon, which is not restricted to rare-gas clusters. The occurrence of the ETMD processes is unambiguously proven by measuring secondary electrons emitted from $\mathrm{LiCl}$ aqueous solution after core ionization of $\mathrm{Li}+($ aq. $)$. Aqueous 
lithium cation is the best test system because this atomic ion has a conveniently simple electronic structure for which ETMD is the only possible electronic relaxation mechanism, facilitating the interpretation of the experimental data. However, ETMD may occur not only here but also in a variety of other solvated metal cations, both after photoionization 29 and as a part of various cascades20.

The experimental results were interpreted with the help of ab initio and molecular dynamics simulations. Our study indicated that contributions of electronic and geometric structures of aqueous solutions are largely separated in the ETMD spectra; an advantage compared to spectra where these contributions are overlaid (for example, X-ray absorption spectra). Indeed, peak positions in the ETMD spectra are determined by the electronic structure and can be reasonably well estimated from the binding energies of participating electrons measured by direct photoemission. Peak shapes and relative intensities, on the other hand, are controlled by the solvation structure.

It is tempting to ask whether the spectroscopy of ETMD electrons can be transformed into a novel tool for structure determination, particularly in aqueous phase. Our theoretical computations revealed that ETMD spectra are sensitive to the immediate environment of the initially ionized lithium ion, reflecting orientations of solvent water molecules, ionwater distances (see Supplementary Figs 3 and 4) and especially ion pairing. There are not many spectroscopic techniques enabling simultaneous access to all this information. Often, due to drawbacks and limitations of individual methods30,31 one has to combine different approaches (for example, X-ray scattering and absorption techniques32) in order to gain comprehensive insight into solvation structure. Although the experimental ETMD spectra reported in the present work have rather poor signal-to-noise ratios, and quantitative analysis of ion pairing is not yet possible, in the theoretical spectra, differences due to distinctive ion pairing situations and geometries of the first solvation shell are well distinguishable. The sensitivity of ETMD spectroscopy stems from the charge-transfer character of the underlying electronic decay processes, which are strongly dependent on molecular overlaps. The molecular overlaps are also the reason for the selective creation of particular electronic states. As demonstrated, electron emission primarily happens from deeper $3 a_{1}$ orbitals of water molecules rather than from the outermost $1 b_{1}$ orbitals. More advanced detection techniques using electron- electron coincidences need to be applied in future studies.

The present work on $\mathrm{Li}_{+}$(aq.) is just a very first step into a new research field. Identification of ETMD in aqueous solutions is relevant for radiation chemistry. Highly reactive slow electrons and water radical cations produced in ETMD should be taken into account in modelling various radiation chemistry processes. With careful tuning of the X-ray photon energy, one can selectively excite an atom, damage its environment while leaving the atom intact, ready for further excitations20. The unique species, lowenergy electrons and radicals created within ETMD are also relevant for the chemistry at biological surfaces and at the electrode-solution interfaces as encountered in many areas of energy-material research. ETMD spectroscopy with its high sensitivity to local structure may become a powerful tool for studying various properties of not only aqueous solutions but also systems with organic or hybrid solvents, for example in Li-ion batteries where the knowledge of ion pairing and local solvation structure is essential for optimization of the ionic conductivity and the energy transfer rate 33 .

\section{Methods}

Experimental. Autoionization electron spectra from 3 and $4.5 \mathrm{M} \mathrm{LiCl}$ aqueous solution were measured from a 15 - $\mu \mathrm{m}$ vacuum liquid-water jet; ionization photon energies were 171 and $175 \mathrm{eV}$, respectively. Experiments were conducted at the U41-PGM undulator beamline of BESSY II, Berlin. The jet velocity was approximately $80 \mathrm{~ms}-1$, and the jet temperature was $6{ }^{\circ} \mathrm{C}$, similar to our previous studies34. Electrons were detected with a hemispherical electron analyser, separated by a $100 \mu \mathrm{m}$ diameter orifice from the liquid jet at a distance of approximately $300 \mu \mathrm{m}$. The two solutions were measured at different times, using different detection geometries. For the $3 \mathrm{MLiCl}$ solution the detection direction was normal with respect to the light polarization vector, whereas for the $4.5 \mathrm{M}$ concentration measurements were performed at the magic angle: approximately $54.7^{\circ}$. Spectra presented in this work were collected over a total time of $120 \mathrm{~min}$, which was broken down into two 60-min data collection periods. This comprises equally long measurements of the solution spectra and of neat-water reference spectra. Longer acquisition times have been attempted. However, slight changes of the liquid-jet position with respect to both the photon beam and the electron detector led to considerable differences in the shape of the distribution of the secondary electrons, which made a meaningful subtraction of pair-wise measured neat-water and solution-spectra impossible. The energy resolution of the U41 beamline was better than $200 \mathrm{meV}$ at the incident photon energies used here, and the resolution of the hemispherical energy analyser is constant with kinetic energy (about $200 \mathrm{meV}$, at $20 \mathrm{eV}$ pass energy). A small X-ray focal size, $23 \times 12 \mu \mathrm{m} 2$, assured that the gas-phase signal amounted to less than $5 \%$ of the total (photo)electron signal. Solutions were prepared by dissolving LiCl (Sigma Aldrich) in highly demineralized water (conductivity $\sim 0.2$ micro Siemens $\mathrm{cm}-1)$.

Computations

Molecular dynamics simulations. We modelled the $\mathrm{LiCl}$ solution using classical molecular dynamics simulations, assuming $3,4.5$ and $6 \mathrm{M} \mathrm{LiCl}$ aqueous solutions. The simulation box for the $3 \mathrm{M}$ solution contained $72 \mathrm{Cl}$ - and $72 \mathrm{Li}+$ ions and 1,259 water molecules in a cubic box with a length of $34.157 \AA$. In the same box size, the more concentrated systems contained $108 \mathrm{Cl}-, 108 \mathrm{Li}+$ ions and 1,235 water molecules (4.5 M); and $144 \mathrm{Cl}-, 144$ $\mathrm{Li}_{+}$ions and 1,211 water molecules $(6 \mathrm{M})$. The force field parameters were taken from ref. 26, the parameters for $\mathrm{Li}+$ were $\sigma=1.80 \AA$ and $\varepsilon=0.07647$ $\mathrm{kJ}$ mol-1. In all simulations, the rigid SPC/E (extended simple point charge model) water model was used 35 . For lithium and chlorine ions, the electronic continuum correction approach 36 was used, yielding scaled charges of +0.75 e and -0.75 e. This approach aims to mimic the effect of electronic polarization in an efficient way; it has been used successfully before for $\mathrm{LiCl}$ solutions25. The simulations runs were $30 \mathrm{~ns}$ long using a time step of $1 \mathrm{fs}$. Simulations were performed at a constant volume and temperature of $300 \mathrm{~K}$ maintained by a CSVR thermostat 37 with a time constant of 0.5 ps. Periodic boundary conditions were employed with short-range electrostatic and van der Waals interactions truncated at $1.2 \mathrm{~nm}$ and the long-range electrostatic interactions treated by the particle mesh Ewald method 38. All simulations were performed with the GROMACS 4.5.3 code39.

Cluster models and geometry optimization. We simulated ETMD spectra for three cluster models, representative of the different ion pairing situations occurring in aqueous solutions: contact, solvent-shared and solvent-separated ion pairs. All clusters consist of one $\mathrm{Li}+$ cation, one $\mathrm{Cl}-$ anion, and five solvent water molecules, but differ in their structural arrangement. Although our cluster models are small, which was necessary in order to make ab initio computations of the ETMD spectra feasible, they nevertheless capture the essential characteristics of the ETMD processes in aqueous solution. Note that ETMD is a charge-transfer process and therefore involves predominantly the nearest neighbours. The Li+cation is fully solvated in all clusters, being surrounded by four water molecules in the first solvation shell in the SShP and SSP configurations. In the CP model, one of the nearest water molecules is substituted by the $\mathrm{Cl}$ - anion. The $\mathrm{Li}_{+}-\mathrm{Cl}$ - distance was set at $2.4 \AA$ in the $\mathrm{CP}$ model, $4.3 \AA$ in the SShP model and $6.4 \AA$ in the $\mathrm{SSP}$ model according to the peak positions in the experimental and theoretical radial distribution functions of the $\mathrm{LiCl}$ aqueous solution. We also fixed the distances between the ions and the nearest water molecules at the values found in the aqueous $\mathrm{LiCl}$ solution, namely $\mathrm{d}\left(\mathrm{Li} \mathrm{i}_{+}-\mathrm{O}\right)=1.95 \AA$ and $\mathrm{d}(\mathrm{Cl}--\mathrm{O})=3.18 \AA 22-$ 
28. With the above constraints, geometry optimization was then performed for each cluster using the second-order Møller-Plesset perturbation theory in conjunction with the $6-311++\mathrm{G}(2 \mathrm{~d}, 2 \mathrm{p})$ basis set.

Simulations of the ETMD spectra. The double-ionization energies of the clusters were calculated using the second-order algebraic diagrammatic construction method, $\mathrm{ADC}(2)$, which is an approximation scheme for the two-particle propagator40,41. In these calculations we employed the Dunning's double- $\zeta$ DZP basis sets 42 for $\mathrm{Li}$ and $\mathrm{Cl}$. The basis set on $\mathrm{Cl}$ was additionally augmented with one s-type and one $\mathrm{p}$-type diffuse functions. Water molecules were described in the same way as in our recent work8, that is, using the cc-pVDZ basis set 43 for hydrogens and a relativistic pseudopotential basis set for oxygens. The latter was augmented with diffuse (one s-type and one p-type) and polarization (one d-type) functions.

The character of each final dicationic ETMD state was determined with the twohole population analysis44. Within this method, the pole strengths of the computed $\mathrm{ADC}(2)$ states are decomposed into contributions originating from configurations with different distributions of the two final outer-valence holes in the system. As these holes may be located either on two different atoms or on the same atom, one distinguishes between 'two-site' and 'one-site' contributions. The one-site contributions are typically used as relative intensities in the simulated decay spectra8. In particular, for constructing the ETMD spectra resulting from $1 \mathrm{~s}$ ionization of $\mathrm{Li}_{+}$, we selected the one-site $\mathrm{Li}$ contributions in the pole strengths. Finally, for comparison with experiment, each spectral line has been convoluted with a Gaussian of full width at half maximum $=3.6 \mathrm{eV}$. This broadening accounts for both the effect of vibrational delocalization occurring during the ETMD processes and for the different solvent configurations present in aqueous solutions.

\section{Acknowledgements}

E.M. and P.S. thank Czech Science Foundation for support (project number 13-34168S). N.V.K. and L.S.C. gratefully acknowledge financial support from the Deutsche Forschungsgemeinschaft. L.S.C also gratefully acknowledges funding from the European Research Council (Advanced Investigator Grant no. 692657). I.U., M.N.P. and B.W. gratefully acknowledge support from the Deutsche Forschungsgemeinschaft (DFG Research Unit FOR 1789). The authors thank the BESSY II staff for assistance during the beamtimes. We thank E. Pluhařová for valuable discussions.

\section{Author contributions}

I.U., R.S., S.T., M.N.P. and B.W. conceived, designed and performed the experiments, and analysed the experimental data. E.M. and P.S. performed and analysed the molecular dynamics simulations. N.V.K. computed the theoretical ETMD spectra and analysed them. B.W., P.S. and N.V.K. co-wrote the paper. All authors discussed the results and commented on the manuscript.

\section{References}

1. Howell, R. W. Auger processes in the 21st century. Int. J. Radiat. Biol. 84, 959-975 (2008).

2. Petit, M. et al. X-ray photolysis to release ligands from caged reagents by an intramolecular antenna sensitive to magnetic resonance imaging. Angew. Chem. Int. Ed. 50, 9708-9711 (2011)

3. Cederbaum, L. S., Zobeley, J. \& Tarantelli, F. Giant intermolecular decay and fragmentation of clusters. Phys. Rev. Lett. 79, 4778-4781 (1997). 4. Averbukh, V. et al. Interatomic electronic decay processes in singly and multiply ionized clusters. J. Electron Spectrosc. Relat. Phenom. 183, 36-47 (2011).

5. Hergenhahn, U. Production of low kinetic energy electrons and energetic ion pairs by intermolecular Coulombic decay. Int. J. Radiat. Biol. 88, 871-883 (2012).

6. Aziz, E. F., Ottosson, N., Faubel, M., Hertel, I. V. \& Winter, B. Interaction between liquid water and hydroxide revealed by core-hole de-excitation. Nature 455, 89-91 (2008).

7. Pokapanich,W. et al. Ionic-charge dependence of the intermolecular Coulombic decay time scale for aqueous ions probed by the core-hole clock. J. Am. Chem. Soc. 133, 13430-13436 (2011).

8. Slavíček, P., Winter, B., Cederbaum, L. S. \& Kryzhevoi, N. V. Proton-transfer mediated enhancement of nonlocal electronic relaxation processes in X-ray irradiated liquid water. J. Am. Chem. Soc. 136, 18170-18176 (2014).

9. Jahnke, T. et al. Ultrafast energy transfer between water molecules. Nat. Phys. 6, 139-142 (2010).

10. Mucke, M. et al. A hitherto unrecognized source of low-energy electrons in water. Nat. Phys. 6, 143-146 (2010).

11. Zobeley, J., Santra, R. \& Cederbaum, L. S. Electronic decay in weakly bound heteroclusters: energy transfer versus electron transfer. J. Chem.

Phys. 115, 5076-5088 (2001)

12. Stumpf, V., Kryzhevoi, N. V., Gokhberg, K. \& Cederbaum, L. S. Enhanced onephoton double ionization of atoms and molecules in an environment of different species. Phys. Rev. Lett. 112, 193001 (2014).

13. LaForge, A. C. et al. Enhanced ionization of embedded clusters by electron-transfer-mediated decay in helium nanodroplets. Phys. Rev. Lett. 116, 203001 (2016)

14. Förstel, M., Mucke, M., Arion, T., Bradshaw, A. M. \& Hergenhahn, U. Autoionization mediated by electron transfer. Phys. Rev. Lett. 106, 33402 (2011)

15. Sakai, K. et al. Electron-transfer-mediated decay and interatomic Coulombic decay from the triply ionized states in argon dimers. Phys. Rev. Lett. 106, 33401 (2011)

16. Yan, S. et al. Observation of interatomic Coulombic decay and electron-transfermediated decay in high-energy electron-impact ionization of Ar2. Phys. Rev. A 88, 42712 (2013).

17. Pernpointner, M., Kryzhevoi, N. V. \& Urbaczek, S. Possible electronic decay channels in the ionization spectra of small clusters composed of Ar and Kr: a four-component relativistic treatment. J. Chem. Phys. 129, 24304 (2008).

18. Stumpf, V., Kolorenč, P., Gokhberg, K. \& Cederbaum, L. S. Efficient pathway to neutralization of multiply charged ions produced in Auger processes. Phys. Rev. Lett. 110, 258302 (2013)

19. Müller, I. B. \& Cederbaum, L. S. Electronic decay following ionization of aqueous Li+ microsolvation clusters. J. Chem. Phys. 122,94305 (2005).

20. Stumpf, V., Gokhberg, K. \& Cederbaum, L. S. The role of metal ions in X-rayinduced photochemistry. Nat. Chem. 8, 237-241 (2016).

21. Weber, R. et al. Photoemission from aqueous alkali-metal-iodide salt solutions using EUV synchrotron radiation. J. Phys. Chem. B 108, 47294736 (2004).

22. Bouazizi, S. \& Nasr, S. Concentration effects on aqueous lithium chloride solutions. Molecular dynamics simulations and X-ray scattering studies. J. Mol. Liq. 197, 77-83 (2014)

23. Petit, L., Vuilleumier, R., Maldivi, P. \& Adamo, C. Ab initio molecular dynamics study of a highly concentrated LiCl aqueous solution. J. Chem. Theory Comput. 4, 1040-1048 (2008).

24. Harsanyi, I. \& Pusztai, L. Hydration structure in concentrated aqueous lithium chloride solutions: a reverse Monte Carlo based combination of molecular dynamics simulations and diffraction data. J. Chem. Phys. 137, 204503 (2012).

25. Pluhařová, E., Fischer, H. E., Mason, P. E. \& Jungwirth, P. Hydration of the chloride ion in concentrated aqueous solutions using neutron scattering and molecular dynamics. Mol. Phys. 112, 1230-1240 (2014).

26. Pluhařová, E., Mason, P. E. \& Jungwirth, P. Ion pairing in aqueous lithium salt solutions with monovalent and divalent counter-anions. J. Phys. Chem. A 117, 11766-11773 (2013).

27. Aragones, J. L., Rovere, M., Vega, C. \& Gallo, P. Computer simulation study of the structure of LiCl aqueous solutions: test of non-standard mixing rules in the ion interaction. J. Phys. Chem. B 118, 7680-7691 (2014).

28. Xu, J.-J., Yi, H.-B., Li, H.-J. \& Chen, Y. Ionic solvation and association in LiCl aqueous solution: a density functional theory, polarised continuum model and molecular dynamics investigation. Mol. Phys. 112, 1710-1723 (2014). 
29. Öhrwall, G. et al. Charge dependence of solvent-mediated intermolecular Coster-Kronig decay dynamics of aqueous ions. J. Phys. Chem. B 114, 17057-17061 (2010).

30. Hefter, G. When spectroscopy fails: the measurement of ion pairing. Pure Appl. Chem. 78, 1571-1586 (2006).

31. van der Vegt, N. F. A. et al. Water-mediated ion pairing: occurrence and relevance. Chem. Rev. 116, 7626-7641 (2016).

32. Näslund, L.-Å. et al. Direct evidence of orbital mixing between water and solvated transition-metal ions: an oxygen 1s XAS and DFT study of aqueous systems. J. Phys. Chem. A 107, 6869-6876 (2003).

33. Jiang, B. et al. The anion effect on Li+ion coordination structure in ethylene carbonate solutions. J. Phys. Chem. Lett. 7, 3554-3559 (2016).

34. Seidel, R., Thürmer, S. \& Winter, B. Photoelectron spectroscopy meets aqueous solution: studies from a vacuum liquid microjet. J. Phys. Chem. Lett. 2, 633-641 (2011).

35. Berendsen, H., Grigera, J. \& Straatsma, T. The missing term in effective pair potentials. J. Phys. Chem. 91, 6269-6271 (1987).

36. Leontyev, I. \& Stuchebrukhov, A. Accounting for electronic polarization in nonpolarizable force fields. Phys. Chem. Chem. Phys. 13, 2613-

2626 (2011).

37. Bussi, G., Donadio, D. \& Parrinello, M. Canonical sampling through velocity rescaling. J. Chem. Phys. 126, 14101 (2007).

38. Essmann, U. et al. A smooth particle mesh Ewald method. J. Chem. Phys. 103, 8577-8593 (1995).

39. Hess, B., Kutzner, C., van der Spoel, D. \& Lindahl, E. GROMACS 4: algorithms for highly efficient, load-balanced, and scalable molecular simulation. J. Chem. Theory Comput. 4, 435-447 (2008).

40. Schirmer, J. \& Barth, A. Higher-order approximations for the particle-particle propagator. Z. Phys. A 317, 267-279 (1984).

41. Velkov, Y., Miteva, T., Sisourat, N. \& Schirmer, J. Intermediate state representation approach to physical properties of dicationic states. J. Chem.

Phys. 135, 154113 (2011).

42. Dunning, T. H. Jr Gaussian basis functions for use in molecular calculations. I. Contraction of (9s5p) atomic basis sets for the first-row atoms. J. Chem. Phys. 53, 2823-2833 (1970).

43. Kendall, R., Dunning, T. \& Harrison, R. Electron affinities of the first-row atoms revisited. Systematic basis sets and wave functions. J. Chem. Phys. 96, 6796-6806 (1992).

44. Tarantelli, F., Sgamellotti, A. \& Cederbaum, L. S. Many dicationic states and two-hole population analysis as a bridge to Auger spectra: strong localization phenomena in BF3. J. Chem. Phys. 94, 523-532 (1991). 